\title{
Growth rates and outcomes of observed large renal masses
}

Naji J. Touma ${ }^{1}$; Gregory W. Hosier ${ }^{1}$; Michael A. Di Lena ${ }^{1}$; Robert J. Leslie ${ }^{1}$; Louisa Ho ${ }^{1}$; Alexandre Menard ${ }^{2}$; D. Robert Siemens ${ }^{1}$

${ }^{1}$ Department of Urology, Queen’s University Kingston, ON, Canada; ${ }^{2}$ Department of Radiology, Queen’s University Kingston, ON, Canada

Cite as: Can Urol Assoc J 2018 December 3; Epub ahead of print. http://dx.doi.org/10.5489/cuaj.5545

Published online December 3, 2018

$* * *$

\section{Abstract}

Introduction: The natural history of small renal masses has been well defined, leading to the recommendation of active surveillance in some patients with limited life expectancy. However, this information is less clear for large renal masses (LRM), leading to ambiguity for management in the older, comorbid patient. The objective of this study was to define the natural history, including the growth rate and metastatic risk, of LRM in order to better counsel patients regarding active surveillance.

Methods: This was a retrospective review of patients with solid renal masses $>4 \mathrm{~cm}$ that had repeated imaging identified from an institutional imaging database. Patient comorbidities and outcomes were obtained through retrospective chart analysis. Outcomes assessed included tumour growth and metastatic rates, as well as cancerspecific (CSS) and overall survival (OS) usimg Kaplan-Meier methodology.

Results: We identified 69 patients between 2005 and 2016 who met the inclusion criteria. Mean age at study entry was 75.5 years; mean tumour maximal dimension at study entry was $5.6 \mathrm{~cm}$. CSS was $83 \%$ and OS $63 \%$ for patients presenting without metastasis, with a mean followup of 57.5 months. The mean growth rate of those that developed metastasis during followup ( $\mathrm{n}=15$ ) was $0.98 \mathrm{~cm} /$ year (95\% confidence interval [CI] 0.33-1.63) as compared to those that did not develop metastasis $(n=46)$, with a growth rate of 0.67 cm/year (95\% CI 0.34-1) (non-significant). Seven patients had evidence of metastasis at the baseline imaging of their LRM and had subsequent growth rate of $1.47 \mathrm{~cm} /$ year $(95 \%$ CI 0.37-2.57) (non-significant)

Conclusions: Compared to small renal masses, LRM are associated with higher metastasis rates and lower CSS and more rapid growth rates. Selection criteria for 
recommending observation of LRM in older, comorbid patients should be more conservative than for small renal masses.

\section{Introduction}

Cancer of the kidney is the third most common urologic malignancy and represents approximately $3.5 \%$ of all malignancies. ${ }^{1}$ The incidence of renal cell carcinoma (RCC) has been rising on average $1.1 \%$ each year over the last 10 years, with this largely attributed to increased frequency of abdominal imaging. ${ }^{2}$ For localized RCC, complete surgical excision by partial or radical nephrectomy remains the gold standard in otherwise healthy patients. However, as more lesions are being incidentally discovered in the elderly and infirm patient populations, competing risks must be balanced when optimizing management. ${ }^{3}$ There has been an increasing interest in the role of active surveillance in select patients with small renal masses, particularly if concurrent illness and surgical morbidity would potentially outweigh benefits of early intervention. ${ }^{4}$

In recent years, a growing body of published data from retrospective and prospective cohort studies have increased our understanding of the clinical, radiographic, and pathological characteristics of untreated renal tumors. Small renal masses, defined as enhancing tumors $\leq 4 \mathrm{~cm}$ in maximal diameter, have emerged as a distinct clinical entity with a growing body of evidence demonstrating their slow radiographic growth and low metastatic potential. ${ }^{5-8}$ A meta-analysis of small renal masses studies revealed a mean growth rate of $0.28 \mathrm{~cm} /$ year. ${ }^{9}$ In a pooled analysis of 936 small renal masses under active surveillance, only 18 patients (1.92\%) developed metastasis. ${ }^{5}$ A prospective nonrandomized study of 497 patients, active surveillance was non-inferior to primary intervention for small renal masses with cancer specific survival of $99 \%$ and $100 \%$ for primary intervention and active surveillance respectively. ${ }^{7}$

While the evidence accumulates for active surveillance as a reasonable management option for T1a kidney cancer in select patients, the growth kinetics and clinical outcomes of untreated larger T1b $(>4 \mathrm{~cm})$ and T2 $(>7 \mathrm{~cm})$ lesions remains poorly characterized. Given the concern for symptomatic progression of these large renal masses (LRM), it is likely that fewer patients are observed on any active surveillance protocol. However, for those older patients with significant concurrent illness, understanding of the growth potential and risk of metastases would be valuable for prognostic purposes and management decisions. The goal of our study was to define the natural history, including the growth kinetics, metastatic risk, and survival of patients with observed LRM.

\section{Methods}

A retrospective search of the radiology imaging database (Nuance Montage, Burlington MS) in a tertiary academic center from 2005 to Jan 2016 was carried out. This is a clinical database with analytics tool for radiology reports. It provides search 
functionality, quality analytics, and business analytics. Search keywords included the following: renal cell carcinoma, renal mass, and renal mass protocol. Excluded in the analysis were clinical stage T1a masses (less than $4 \mathrm{~cm}$ ), and masses with appearance suspicious for angiomyolipoma, renal cystic disease with a Bosniak 2F classification or less, non-renal metastatic disease and urothelial cell carcinoma. Enhancing renal masses measuring greater than $4 \mathrm{~cm}$ concerning for renal cell carcinoma with at least 2 crosssectional imaging studies at least 6 months apart were included in the analysis. Given the limitations with measurements, ultrasounds were not included in this study. Three dimensional measurements of each LRM were re-assessed by a single radiologist. It is important to highlight that this was not a surveillance cohort. These are patients with renal masses suspicious for renal cell carcinoma who have had at least 2 cross sectional imaging studies 6 months apart. Some were never seen in a urology clinic. Some were seen, but were deemed inoperable. Some were consented for surgery but may have waited at least 6 months for surgery or may have had surgery after a period of observation because of clinical/radiological progression. It is a comprehensive way to look at the natural history of all these masses inside and outside of urological care. Examined covariates included patient age at diagnosis, sex, tumor size at presentation, and evidence of metastatic kidney cancer at presentation. Other covariates recorded included American Society of Anesthesiologists (ASA) score, baseline creatinine, platelets, hypertension, diabetes, coronary artery disease, and hypercholesterolemia. The primary outcome of interest was growth rate of maximal tumor diameter (defined as the net change in linear diameter per year). Size comparisons were made by a single radiologist using a consistent radiographic characteristic (maximum tumor diameter). Care was taken to ensure a similar cross-sectional cut from which the data were obtained when comparing interval growth where possible. The growth rates calculations were censored once metastatic patients were placed on systemic targeted therapy or at the time of surgical extirpation. Other outcomes of interest included progression to metastatic disease, cancer-specific survival (CSS) and overall survival (OS). For these secondary outcomes, censorship did not occur at the time of the last available imaging making follow-up much longer.

\section{Statistical analysis}

Student's T and Fisher's exact tests were used for continuous and categorical variables respectively. Kaplan-Meier curves were created for metastasis-free, cancer-specific, and overall survival using GraphPad Prism 7. Results were regarded as statistically significant at a p value of 0.05 . 


\section{Results}

Search results of the institutional imaging database included 2204 discrete cases that met the key search terms. After application of the exclusion criteria, 69 patients were found to have LRM consistent with renal cell carcinoma (RCC) and had repeated imaging consistent with at least short-term observation of 6 months (See Figure 1). Patient demographics for the 69 cases are depicted in Table 1. Mean age at study entry was 75.5 years (range 56.4-92.1). Mean follow-up for this primary outcome of growth rate was 28.4 months (range 6-118). Mean follow-up of survival outcomes was much longer at 57.5 months. After abstraction from the patient charts, it was determined that the majority of patients ( $\mathrm{n}=51 ; 73.9 \%)$ had their LRM identified incidentally while asymptomatic. Mean initial tumor size at presentation was $5.7 \mathrm{~cm}$ (range $4-17.6 \mathrm{~cm}$ ). Of the 69 patients, 21 (30.9\%) had biopsies either of their renal lesion or of a metastatic deposit, all of which confirmed RCC. Out of the entire cohort, 15 (22.1\%) patients developed metastases during follow-up and 8 (11.6\%) had evidence of metastatic disease at the time of their baseline renal imaging. Mean age, renal function, and follow-up time were not different between those with metastasis and those never developing metastasis (Table 1). Mean tumor size at presentation was higher for metastatic cases compared to non-metastatic cases (6.5 vs. $5.25 \mathrm{~cm}, \mathrm{p}=0.024)$. Of the entire cohort, 10 (22\%) and 2 (4\%) patients progressed to radical and partial nephrectomy respectively after an initial period of observation.

The overall growth rate of these monitored LRM was $0.82 \mathrm{~cm} /$ year $(95 \%$ confidence interval [CI] 0.55-1.09). In patients who did not develop metastasis during the follow-up period, growth rate was $0.67 \mathrm{~cm}$ /year (95\% CI 0.34-1). Growth rate was 0.98 $\mathrm{cm} /$ year (95\% CI 0.33-1.67) in patients who progressed to metastatic disease and 1.14 $\mathrm{cm} /$ year (95\% CI 0.37-2.57) in patients who had metastasis at presentation (NS) (Figure 2).

CSS and OS of the entire cohort was 75\% and 50\% respectively over the followup period of 57.5 months. For patients without metastasis at presentation, CSS and OS were $83 \%$ and $63 \%$ over the same follow-up. Mean overall survival was 90.6 months (95\% CI 71.2 - 110) with a mean cancer specific survival of 127.6 months (95\% CI 98.1

- 157.1; Figure 2). Of those 61 patients without metastasis at presentation, mean metastatic-free survival was 131.9 months (CI 101.0 - 162.8; Figure 3).

\section{Discussion}

The natural history of small renal masses $<4 \mathrm{~cm}$ has been well defined leading to the recommendation of considering active surveillance in patients with diminished life expectancy. ${ }^{10}$ Less clear, however, is the management of older patients with LRM, particularly those with significant co-morbid illness. Herein, we report the growth rates, metastatic rates, CSS and OS in the largest cohort of patients with LRM suspicious for RCC $>4 \mathrm{~cm}$ to date. The main findings of this study are that LRM appear to be 
associated with higher metastasis rates and lower cancer-specific survival compared to contemporary series of patients with small renal mass. In addition, the growth rates of LRM appear to be more rapid than small renal masses. ${ }^{9}$

In this present study cohort, we included 8 patients (11.6\%) that had evidence of metastatic disease at the time of their baseline imaging as an interesting comparison for those co-morbid patients undergoing surveillance without metastases at presentation. From this cohort without evidence of metastatic disease, 15 patients (24\%) subsequently developed metastases during observation. In a pooled analysis of 936 small renal masses under active surveillance, only 18 patients (1.92\%) developed metastasis. ${ }^{5}$ CSS and OS were $83 \%$ and $63 \%$ respectively for patients without metastasis at presentation. By contrast, CSS and OS in patients with small renal masses were 100\% and 75\% respectively as reported in The Delayed Intervention and Surveillance for Small Renal Masses (DISSRM) Registry. ${ }^{7}$ As renal mass size increases, the risk of the mass representing a malignancy increases as well. Pathological analysis of 2935 surgically excised renal masses showed a mean size of $6.3 \mathrm{~cm}$ for malignant tumors and $4.2 \mathrm{~cm}$ for benign tumors. ${ }^{11}$ The proportion of high grade malignancies also increases with increasing renal mass size as $57.7 \%$ of RCC tumors $>7 \mathrm{~cm}$ were high grade compared to only $2.3 \%$ of renal masses $<1 \mathrm{~cm}$. ${ }^{11}$ Taken together, the increased risk of malignant high grade tumors in larger renal masses is in line with our findings of increased metastatic rates and decreased cancer-specific survival for LRM compared to those reported for renal masses $<4 \mathrm{~cm}$.

Compared to other studies for LRM, the present rates of metastasis were higher and rates of CSS were lower. In the three previously reported cohorts of patients with LRM $>4 \mathrm{~cm}$, metastatic rates were $0-5.6 \%$ compared to our metastatic rate of $24 \%{ }^{12-}$ ${ }^{14} \mathrm{CSS}$ in each of these cohorts over a follow-up period of $24-36$ months was $100 \%$ compared to $83 \%$ in our study. It is possible that methodological differences in study population selection contributed to our increased rates of metastasis and decreased CSS. Although the mean patient age, renal mass size, and rates of progression to surgical intervention were similar to other large renal mass cohorts, the mean growth rate of masses in the present study was higher than all three prior cohorts $(0.82 \mathrm{~cm} /$ year compared to $0.39-0.57 \mathrm{~cm} /$ year). ${ }^{12-14}$ Most evidently, in contrast to these prior studies we included patients with metastasis at presentation as an interesting comparator. The tumor growth rate in this group was $1.14 \mathrm{~cm} /$ year compared to $0.67 \mathrm{~cm} /$ year and $0.98 \mathrm{~cm} /$ year for patients without metastasis and those who went on to develop metastasis respectively.

Beyond including patients with apparent metastatic disease at their baseline imaging, other methodological differences in selecting this study population may have contributed to the differences compared to prior studies of LRM. This study cohort incorporated all patients with documented renal masses concerning for RCC captured in an imaging database that were observed at least over a 6-month interval. In contrast, 
previous investigations of LRM included patients that were identified and selected from clinical datasets and it is possible that these were biased by the fact that they were referred to urological specialists for management. Indeed, the majority of patients in the study had significant co-morbidity and low OS, indicating a high-risk surgical population that may not have necessarily been referred for surgical consultation.

LRM growth rates in patients, who did not develop metastasis was $0.67 \mathrm{~cm} /$ year as compared to those that had metastases at presentation $(1.14 \mathrm{~cm} /$ year $)$ and those that went on to develop metastasis on observation (0.98 cm/year). (NS) The evidence for growth rates as a risk factor for development of metastasis is somewhat mixed. In a pooled analysis of the small renal mass literature, Smaldone et al. found that the growth rate for masses that would go on to metastasize was $0.8 \mathrm{~cm} /$ year compared to $0.3 \mathrm{~cm} /$ year for masses without metastasis. ${ }^{5}$ Additionally, there were a proportion of renal masses that exhibited no growth over the study period of which none developed metastasis. ${ }^{5}$

Additionally, growth rates $>0.5 \mathrm{~cm}$ /year have been found to correlate with high grade in clear cell carcinoma, thereby inferring that fast growth rates may result in poorer prognosis. ${ }^{15}$ In contrast, growth rates between oncocytoma and RCC have been shown to be similar with minimal difference in malignancy rates of growing versus non-growing masses. ${ }^{16,17}$ In addition, the Renal Cell Carcinoma Consortium of Canada found no association between growth rates and progression to metastatic disease. ${ }^{18,19}$ The DISSRM registry found that there is great variability in the growth kinetics of small renal masses early in active surveillance (initial 6-12 months), but the variability and growth rates

decrease over time. ${ }^{20}$ Nevertheless, growth rate is the main trigger for intervention in small renal mass surveillance protocols. The present study is the first to compare growth kinetics among patients with and without metastases in a LRM cohort and it remains to be confirmed whether parameters such as growth rates are predictive of outcomes in other LRM cohorts.

Due to the retrospective nature of this study, there are several limitations to address. First, it is not possible to discern if all patient factors were fully and accurately recorded. Second, there was no initial imaging protocol to determine presence of metastasis at presentation. It would therefore be possible that a proportion of patients had metastasis at initial presentation that was only identified later. However, this methodology was not different than other reports of large renal mass cohorts and would not necessarily explain our higher rates of metastasis.

\section{Conclusion}

Compared to small renal masses, the growth rate of LRM in this study was more rapid and associated with higher rates of metastases and lower CSS. Recommendations of observation, even for older, co-morbid patients, should be made with caution given the worse outcomes of LRM compared to those with small renal masses. 


\section{References}

1. Siegel R.L., Miller K.D., Jemal A. Cancer statistics, 2016. Cancer J Clin. 2016;66:7

2. Howlader N., Noone A., Krapcho M. et al. SEER Cancer Statistics Review, 19752013, National Cancer Institute. Natl Cancer Inst. 2016

3. Hollingsworth J.M., Miller D.C., Daignault S. et al. Rising incidence of small renal masses: A need to reassess treatment effect. JNCI J Natl Cancer Inst. 2006;98:1331

4. Jewett M., Rendon R., Lacombe L., et al. Canadian guidelines for the management of small renal masses (SRM). Can Urol Assoc J. 2015;9:160

5. Smaldone M.C., Kutikov A., Egleston B.L. et al. Small renal masses progressing to metastases under active surveillance. Cancer. 2012;118:997

6. Organ M., Jewett M., Basiuk J. et al. Growth kinetics of small renal masses: A prospective analysis from the Renal Cell Carcinoma Consortium of Canada. Can Urol Assoc J. 2014;8:24

7. Pierorazio P.M., Johnson M.H., Ball M.W. et al. Five-year analysis of a multiinstitutional prospective clinical trial of delayed intervention and surveillance for small renal masses: The DISSRM Registry. Eur Urol. 2015;68:408

8. Uzosike A.C., Patel H.D., Alam R. et al. Growth kinetics of small renal masses on active surveillance: Variability and results from the DISSRM Registry. J Urol. 2017;199:641

9. Chawla S.N., Crispen P.L., Hanlon A.L. et al. The natural history of observed enhancing renal masses: meta-analysis and review of the world literature. J Urol. 2006; $175: 425$

10. Campbell S., Uzzo R.G., Allaf M.E. et al. Renal mass and localized renal cancer: AUA Guideline. J Urol. 2017;198:520

11. Frank I., Blute M.L., Cheville J.C. et al. Solid renal tumors: An analysis of pathological features related to tumor size. J Urol. 2003;170:2217

12. Lamb G.W.A., Bromwich E.J., Vasey P. et al Management of renal masses in patients medically unsuitable for nephrectomy--natural history, complications, and outcome. Urology. 2004;64:909

13. Mues A.C., Haramis G., Badani K. et al. Active surveillance for larger (cT1bN0M0 and cT2N0M0) renal cortical neoplasms. Urology. 2010;76:620

14. Mehrazin R., Smaldone M.C., Kutikov A. et al. Growth kinetics and short-term outcomes of cT1b and cT2 renal masses under active surveillance. J Urol. 2014;192:659 
15. Zhang L., Yin W., Yao L. et al: Growth pattern of clear cell renal carcinoma in patients with delayed surgical intervention: fast growth rate correlates with high grade and may result in poor prognosis. Biomed Res Int. 2015; 2015: 598134

16. Siu W., Hafez K.S., Johnston W.K. et al. Growth rates of renal cell carcinoma and oncocytoma under surveillance are similar. Urol Oncol Semin Orig Investig. 2007;25:115

17. Kunkle D.A., Crispen P.L., Chen D.Y.T. et al. Enhancing renal masses with zero net growth during active surveillance. J Urol. 2007;177:849

18. Mason R.J., Abdolell M., Trottier G. et al. Growth kinetics of renal masses: analysis of a prospective cohort of patients undergoing active surveillance. Eur Urol. 2011; 59:863

19. Organ M., Jewett M., Basiuk J. et al. Growth kinetics of small renal masses: a prospective analysis from the Renal Cell Carcinoma Consortium of Canada. Can Urol Assoc J. 2014; 8:24

20. Uzosike A.C., Patel H.D., Alam R. et al. Growth kinetics of small renal masses on active surveillance: variability and results from the DISSRM registry. $J$ Urol. 2018;199:641 


\section{Figures and Tables}

Fig. 1. Determination of study population.

Renal mass images screened $(n=2204)$<smiles>C[12CH2]</smiles>

Individual patients $(\mathrm{n}=1128)$

Exclusion criteria:

-T1a masses $(n=1019)$

-renal mass appearance not consistent

with RCC (ex: AML...) $(\mathrm{n}=12)$

-Fewer than 2 repeat images

$>6$ months apart $(n=28)$

Final study population $(\mathrm{n}=69)$

Fig. 2. Growth rate of large renal masses in patients with no metastasis, who went on to develop metastasis and had metastasis at presentation.

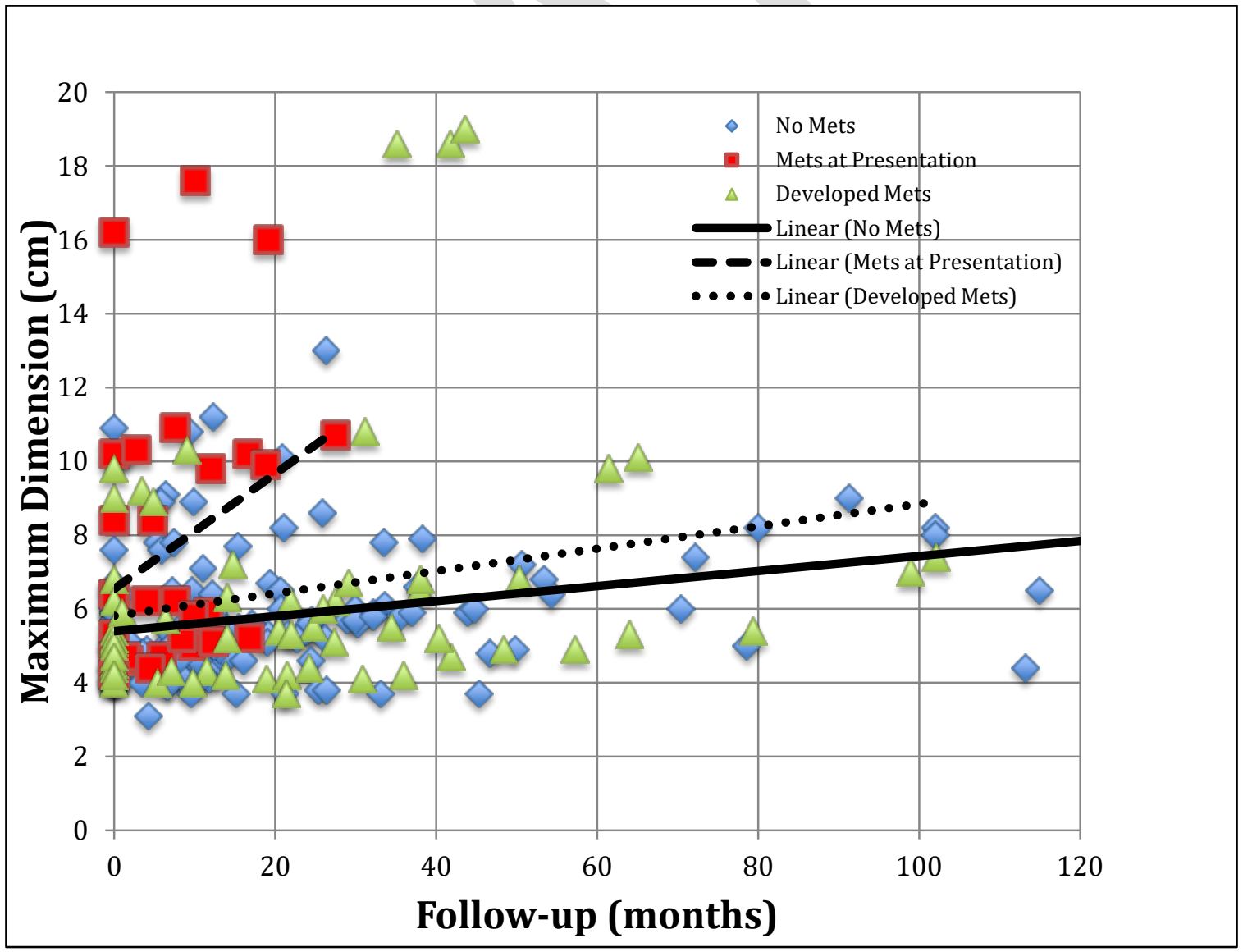


Fig. 3. Kaplan-Meier curves for (A) metastasis-free survival; (B) cancer-free survival; and $(\boldsymbol{C})$ overall survival.
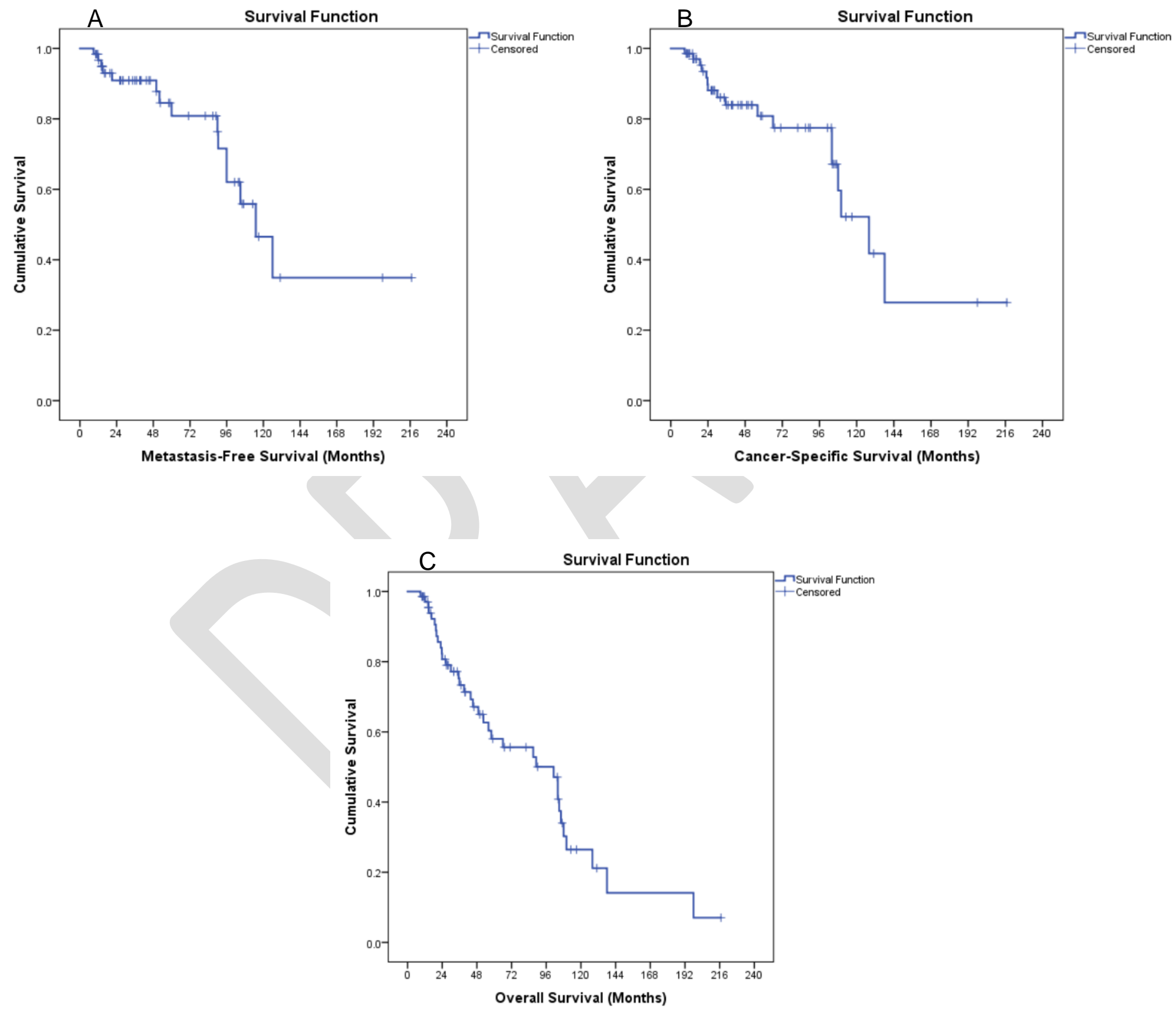


\begin{tabular}{|c|c|c|c|c|}
\hline Characteristic & $\begin{array}{c}\text { Overall } \\
N=69\end{array}$ & $\begin{array}{c}\text { No mets } \\
\mathrm{N}=46\end{array}$ & $\begin{array}{c}\text { Mets } \\
\text { N=23 }\end{array}$ & $\mathbf{p}$ \\
\hline & \multicolumn{4}{|c|}{ Mean (SD) } \\
\hline Age years (range 56-92) & $75.5(8)$ & $76.6(8.3)$ & $73.2(6.8)$ & 0.1023 \\
\hline eGFR, $\mathrm{ml} / \mathrm{min} / 1.73 \mathrm{~m}^{2}$ (range $6->120$ ) & $57.5(23)$ & $60.4(20.3)$ & $51(27.6)$ & 0.14 \\
\hline Tumour size, cm (range 4-17.6) & $5.7(2.2)$ & $5.25(1.41)$ & $6.5(3.1)$ & 0.024 \\
\hline Growth rate, cm/year (range 0.33-2.57) & $0.82(1.2)$ & $0.67(1.1)$ & $1.13(1.2)$ & 0.12 \\
\hline \multirow[t]{2}{*}{ Followup, months (range 6-118) } & $28.4(28)$ & $30.4(32)$ & $24.8(21.7)$ & 0.44 \\
\hline & \multicolumn{4}{|c|}{$\mathbf{n}(\%)$} \\
\hline Male & $40(58)$ & $28(60)$ & $12(52)$ & 0.60 \\
\hline ASA & $2=$ & & & - \\
\hline 1 & $0(0)$ & $0(0)$ & $0(0)$ & \\
\hline 2 & $10(15)$ & $9(20)$ & $1(3)$ & 0.15 \\
\hline 3 & $37(54)$ & $24(52)$ & $13(57)$ & 0.80 \\
\hline 4 & $21(31)$ & $12(26)$ & $9(40)$ & 0.28 \\
\hline Smoker & $38(55)$ & $26(57)$ & $12(52)$ & 0.80 \\
\hline Solitary kidney & $4(6)$ & $1(2)$ & $3(13)$ & 0.10 \\
\hline Symptomatic & $18(26)$ & $10(22)$ & $8(35)$ & 0.80 \\
\hline Stage & & & & \\
\hline T1b & $60(87)$ & $43(94)$ & $17(74)$ & 0.051 \\
\hline T2a & $4(6)$ & $1(2)$ & $3(13)$ & 0.104 \\
\hline T2b & $5(7)$ & $2(4)$ & $3(13)$ & 0.32 \\
\hline Biopsy $^{*}$ & $21(31)$ & $10(22)$ & $11(48)$ & 0.0501 \\
\hline Intervention & S & & & \\
\hline Radical nephrectomy & $10(15)$ & $10(22)$ & $0(0)$ & 0.025 \\
\hline Partial nephrectomy & $2(3)$ & $2(4)$ & $0(0)$ & 0.55 \\
\hline Embolization & $1(1)$ & $0(0)$ & $1(4)$ & 0.33 \\
\hline Pathology & 28 & 17 & & \\
\hline Clear-cell & $23(82)$ & $12(71)$ & $11(100)$ & 0.104 \\
\hline Undifferentiated & $3(11)$ & $3(18)$ & $0(0)$ & 0.55 \\
\hline Papillary & $1(3.5)$ & $1(5.5)$ & $0(0)$ & 1.0 \\
\hline Multilocular cystic & $1(3.5)$ & $1(5.5)$ & $0(0)$ & 1.0 \\
\hline Overall mortality & $35(51)$ & $17(37)$ & $18(78)$ & 0.002 \\
\hline Cancer-specific mortality & $17(25)$ & $8(17)$ & $9(40)$ & 0.07 \\
\hline
\end{tabular}

*Kidney or metastatic site. ASA: American Society of Anesthesiologists; eGFR: estimated glomerular filtration rate; SD: standard deviation. 